\title{
Risk factors and prognostic value of daytime Cheyne-Stokes respiration in chronic heart failure patients
}

\author{
Roberta Poletti ${ }^{\text {a }}$, Claudio Passino ${ }^{\mathrm{a}, \mathrm{b}}$, Alberto Giannoni ${ }^{\mathrm{a}}$, Luc Zyw ${ }^{\mathrm{a}}$, Concetta Prontera ${ }^{\mathrm{a}}$, \\ Francesca Bramanti ${ }^{\mathrm{a}}$, Aldo Clerico ${ }^{\mathrm{a}, \mathrm{b}}$, Massimo Piepoli ${ }^{\mathrm{c}}$, Michele Emdin ${ }^{\mathrm{a}, *}$ \\ ${ }^{a}$ Cardiovascular Medicine Department and Cardiovascular Endocrinology Laboratory, Foundation G. Monasterio, CNR-Regione Toscana, Pisa, Italy \\ ${ }^{\mathrm{b}}$ Scuola Superiore S. Anna, Pisa, Italy \\ ${ }^{\mathrm{c}}$ Cardiology Department, G. da Saliceto Polichirurgico Hospital, Piacenza, Italy
}

Received 23 October 2007; received in revised form 8 June 2008; accepted 28 June 2008

Available online 8 August 2008

\begin{abstract}
Background: Sleep-related Cheyne-Stokes (CS) respiration is a known phenomenon in chronic heart failure (CHF). We aimed to study the prevalence, clinical correlates, risk factors and prognostic relevance of daytime CS, as well as its relation with neurohormonal derangement. Methods: One hundred forty seven CHF patients with left ventricular systolic dysfunction (age: $64 \pm 12$ years, ejection fraction, EF, $31 \pm 8 \%$, mean $\pm \mathrm{SD}$ ) underwent morning polygraphic recording, in addition to comprehensive clinical and neurohormonal evaluation.

Results: Daytime CS was detected in 87 patients (59\%), and associated with worse NYHA class $(2.6 \pm 0.7$ vs $2.2 \pm 0.8, P<0.05)$, lower EF $(29 \pm$ 8 vs $33 \pm 8 \%, P<0.05)$, peak oxygen consumption $(11.3 \pm 8.3 \mathrm{vs} 13.4 \pm 4 \mathrm{~mL} / \mathrm{min} / \mathrm{kg}, P<0.05)$, resting carbon dioxide level (33.1 $\pm 4.2 \mathrm{vs} 37.9 \pm$ $3.8 \mathrm{~mm} \mathrm{Hg}, P<0.001)$, higher norepinephrine [588 (395-939) vs (331-681) ng/L, median (interquartile range) $P<0.01$ ] and natriuretic peptides [ANP: 136 (57-230) vs 66 (18-103); BNP: 284 (99-510) vs 64 (21-202); NT-proBNP: 2575 (814-3320) vs 448 (147-1599) ng/L, all: $P<0.001]$. At univariate analysis, $\mathrm{CS}$ risk factors were age, $\mathrm{EF}$, carbon dioxide, creatinine, norepinephrine, natriuretic peptides, whereas age and NT-proBNP level were the only multivariate predictors. On a 33-month follow-up, CS resulted among univariate predictors of cardiac death, NT-proBNP emerging as the only variable at multivariate analysis.

Conclusions: Daytime CS is frequent in CHF and is correlated with clinical severity, neurohormonal derangement, particularly of NTproBNP, and long-term prognosis.

(C) 2008 Elsevier Ireland Ltd. All rights reserved.
\end{abstract}

Keywords: Cheyne-Stokes respiration; Natriuretic peptides; Norepinephrine; Heart failure; Prognosis

\section{Introduction}

Periodic breathing frequently occurs in patients with chronic heart failure (CHF), and is characterized by Cheyne-Stokes respiration (CS) either with central hypopneas or apneas alternating with hyperventilation [1]. CS has been

\footnotetext{
* Corresponding author. G. Monasterio Foundation, CNR-Regione Toscana, Via Giuseppe Moruzzi 1, 56124 Pisa, Italy. Tel.: +39 0503152189; fax: +390503152109 .

E-mail address: emdin@ifc.cnr.it (M. Emdin).
}

more commonly detected at nighttime as sleep-related, in association with oxyhemoglobin desaturation, frequent arousals, sleep fragmentation [2], altered quality of life [3], and sympathetic overactivation [4-5], which may contribute to deterioration of cardiac function and reduced survival $[6,7]$. Few studies in small subsets have evaluated CS at daytime $[4,8]$ in awake patients too, indicating a poor prognosis [9].

Neurohormonal derangement, involving activation of sympathetic, renin-angiotensin-aldosterone systems, and cardiac natriuretic peptide systems is a hallmark of CHF [10-11]. Plasma levels of brain natriuretic peptide (BNP) 
and of amino-terminal fragment of probrain natriuretic hormone (NT-proBNP), in particular, have been recognized as diagnostic and prognostic markers in CHF patients [12].

Whereas determinants of central sleep apneas have already been described [13], risk factors and clinical correlates of daytime CS remain unknown. Furthermore, the possible association of daytime CS and neurohormonal activation system has not been fully investigated. The aim of the present study was to evaluate prevalence and risk factors for daytime CS in CHF patients.

\section{Methods}

\subsection{Patients}

From September 2002 to November 2005, we selected 184 consecutive CHF patients with depressed left ventricular ejection fraction (EF, $<45 \%$ ) at echocardiography. Exclusion criteria were acute coronary syndrome within the preceding 6 months, severe chronic obstructive pulmonary disease, severe renal impairment (serum creatinine level $>2.5 \mathrm{mg} / \mathrm{dl}$ ), use of morphine, benzodiazepines, any psychotropic drug or theophilline derivatives. In order to focus on central hypopneas and apneas, additional exclusion criteria were also chosen, such as obesity (body mass index $>30 \mathrm{~kg} / \mathrm{m}^{2}$ ) and history of snoring and upper airways obstruction. Of the 184 patients, 37 did not fulfill these criteria, leaving 147 patients for enrollment [(118 males and 29 females, mean age $64 \pm 12$ years, body mass index $25 \pm 2.7 \mathrm{~kg} / \mathrm{m}^{2}$, EF $31 \pm$ $8 \%$, with ischaemic $(46 \%)$, idiopathic $(39 \%)$ or secondary $(15 \%)$ cardiomyopathy ( $8 \%$ with hypertensive cardiomyopathy, and $7 \%$ with valvular disease), NYHA class I-II $65 \%$, III-IV 35\%)]. All patients were on optimal pharmacological treatment (82\%: furosemide, 73\%: carvedilol/bisoprolol, 84\%: angiotensin-converting enzyme inhibitors/angiotensin-receptor blockers, 59\%: spironolactone); 12 patients (9\%) had previously received cardiac resynchronisation therapy.

The study was approved by the Institute's Ethical Committee and informed consent was obtained from all patients.

\subsection{Cardiopulmonary exercise test, echocardiographic study and plasma assays}

A symptom-limited cardiopulmonary test was performed on a bicycle ergometer using a ramp protocol with increments of $10 \mathrm{~W} / \mathrm{min}$ (Vmax, Sensormedics, USA). All echocardiographic studies were performed by the same physician. Blood samples were drawn at 8 a.m. from an antecubital vein, after a 20-minute supine rest. Atrial natriuretic peptide (ANP), BNP, plasma catecholamines, plasma renin activity (PRA), aldosterone, and thyroid hormones were assayed as described elsewhere [10]; NTproBNP was measured with an automated electrochemiluminescent immunoassay (Roche diagnostics, Germany).

\subsection{Polygraphy}

During the same morning, all subjects, in the supine position, were asked to relax without falling asleep, and during spontaneous breathing were evaluated for a 20minute period, with a previously validated technique [4]. During each examination, we recorded a II electrocardiographic lead, chest and abdominal movement by electrical inductance, oronasal airflow, beat-to-beat blood pressure (Colin ${ }^{\circledR}$ tonometry, San Antonio, TX, USA), oxygen saturation (Pulse Oxymeter Pulsox-7, Minolta ${ }^{\circledR}$ ) and endtidal pCO2 signal $\left(\mathrm{PETCO}_{2}, \mathrm{Cosmoplus}^{\circledR}\right.$, Novametrics). Data were digitized (300 sample/s), and stored on a personal computer. CS was defined either by the occurrence of cyclic episodes of apnea (cessation of flow and respiratory movements for $\geq 10 \mathrm{~s}$ ) [5] or of hypopnea (reduction in the tidal volume greater than $50 \%$, lasting $\geq 10 \mathrm{~s}$, followed by a reduction in oxygen saturation of $\geq 4 \%$ ), followed by hyperpnoea.

Fifty-three patients (36\%) also underwent, within $24 \mathrm{~h}$, a nocturnal continuous polysomnographic recording. An apnea-hypopnea index (defined as the number of apneas and hypopneas per hour) $\geq 20$ was used as arbitrary cut-off to define relevant nighttime CS. Main clinical characteristics of patients receiving nocturnal recording were similar to those of the overall population: 41 males and 12 females, mean age $65 \pm 1$ years, body mass index $25 \pm 0.3 \mathrm{~kg} / \mathrm{m}^{2}$, EF $31 \pm 1 \%$, with ischaemic (44\%), idiopathic $(38 \%)$ or secondary (18\%) cardiomyopathy, NYHA class I-II 63\%, III-IV $37 \%$.

\subsection{Follow-up}

Follow-up started at the time of hospital admission and continued until study termination. Independent interviewers obtained information directly from patients, relatives, or Institute cardiologists or general practitioners, regarding the date of death, between the time of discharge and September 2007. The primary end-point was cardiac death, including pump failure (defined as a death resulting from multiorgan failure caused by heart failure progression) and sudden death (defined as a witnessed cardiac arrest, death within $1 \mathrm{~h}$ after the onset of acute symptoms, or an unexpected death in a patient known to have been well within the previous $24 \mathrm{~h}$ ). Information about time and cause of death was obtained from death certificates, post-mortem reports and family doctors. The individual follow-up ended with death or with cardiac surgery (ventricular assist device implantation, heart transplantation). No patient was lost to follow-up.

\subsection{Statistical analysis}

Statistical analysis was carried out using SPSS 12.0 (SPSS inc., Chicago, Il, USA). Due to the skewed distribution of BNP, NT-proBNP, norepinephrine, PRA and aldosterone, their natural logarithm was used for statistical 

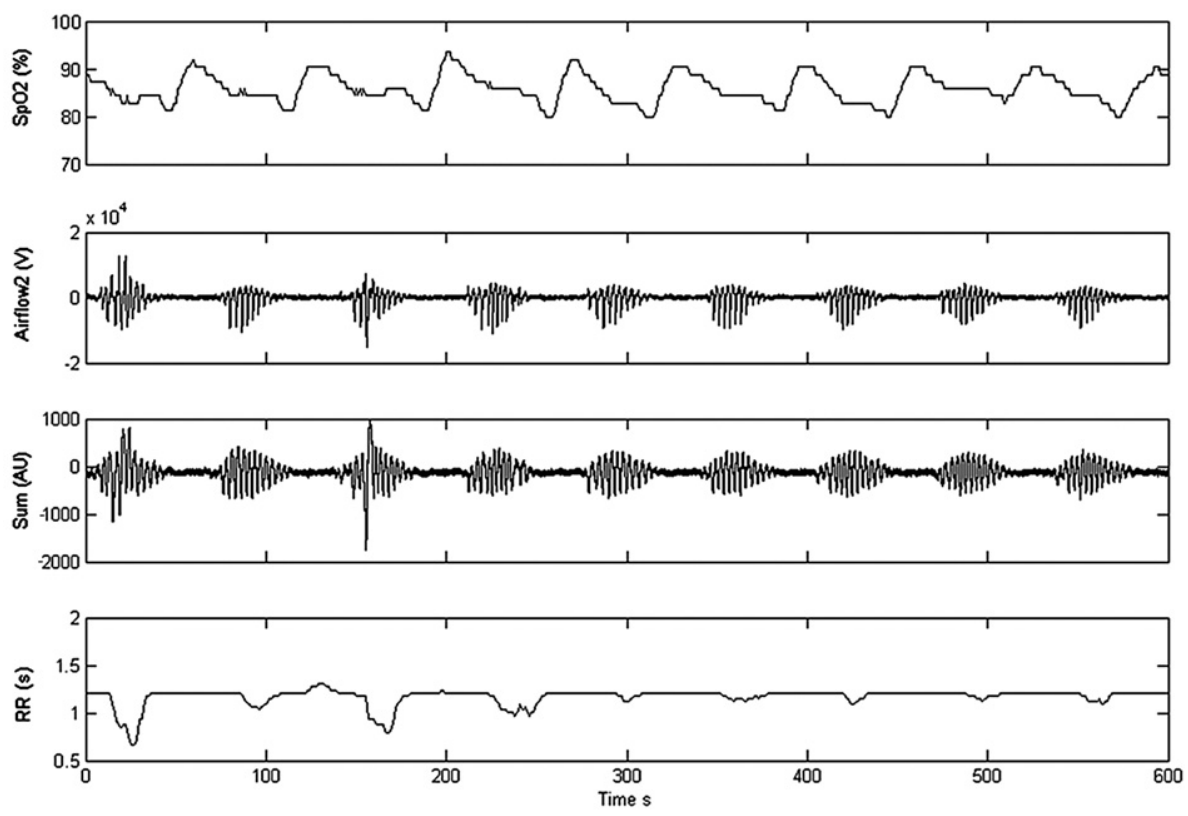

Fig. 1. Top-down: Time-course of oxygen saturation (SpO2), airflow, combined respiratory signal (Sum) from chest and abdominal inductance recordings, RR interval (RR) in a 62-year old patient with ischaemic cardiomyopathy and daytime Cheyne-Stokes respiration are shown.

analysis. The same principle was applied for other markers, when needed. Groups were compared for frequencies or categorical data (i.e., NYHA class) using the chi-squared test. Differences between groups in continuous variables were evaluated by the $t$-test for independent samples; differences between groups in categorical variables were evaluated by chi-square or Fischer exact test. Univariate and multiple logistic regression analyses were used to examine the association between several baseline clinical and neurohormonal variables and the presence of daytime CS occurrence. The candidate independent variables used for analysing risk factors for CS were selected on the basis of the strength of association with CS presence shown by previous studies in similar populations: body mass index, NYHA functional class, EF, presence of atrial fibrillation, presence of a paced cardiac rhythm, age, gender, mean resting $\mathrm{PETCO}_{2}$ during wakefulness, medication, and cause of CHF, as previously described for sleep-related CS [13]. In addition, we have considered serum creatinine level, arterial pressure, left atrium area, maximum workload and peak oxygen consumption at cardiopulmonary test, plasma level of ANP, BNP, NTproBNP, norepinephrine, renin activity, and aldosterone. Univariate predictors (age, ejection fraction, serum creatinine, NYHA functional class, peak oxygen consumption at cardiopulmonary test, plasma level of BNP, NT-proBNP and norepinephrine) were then considered for multivariate Cox proportional hazard regression analysis, with the exception of plasma BNP which, although closely related to NT-proBNP secretion, had a lower level of significance.

The predictive power of variables was quantified using receiver operating characteristic curves (ROC). A difference in the area under the curve (AUC) defined the increment in predictive power between different models. The statistical significance of differences in AUC from the line of "no information" and between different curves was evaluated with Mann-Whitney $U$-Statistics.

Survival curves were analysed using the Kaplan-Meier estimate and comparisons were made with log-rank test. In addition, Cox proportional hazards model was used to identify significant prognostic. The relative risk for each independent variable in the hazard equation was directly proportional to the risk brought by that variable to the model. All hazard ratios are presented with $95 \%$ confidence intervals and all $P$ values are two-sided.

The candidate variables chosen for survival analysis have already been linked to long-term survival in previous studies conducted in similar populations: presence of daytime CS, NYHA functional class, EF, presence of atrial fibrillation, body mass index, age, gender, medication, serum creatinine level, peak oxygen consumption at cardiopulmonary test,

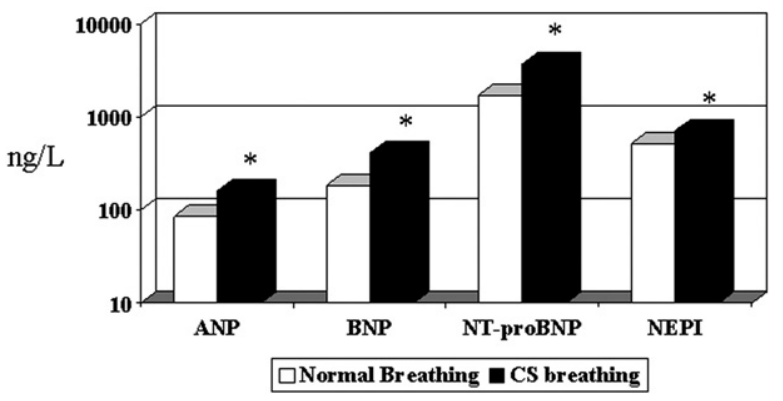

Fig. 2. Comparison of plasma concentration of atrial natriuretic peptide (ANP), brain natriuretic peptide (BNP), amino-terminal fragment of probrain natriuretic peptide (NT-proBNP), and norepinephrine (NEPI) in patients with Cheyne-Stokes respiration (CS) and patients with normal breathing; ${ }^{*} P<0.01$. 
Table 1

General characteristics and neurohormonal findings in patients with normal breathing pattern and with Cheyne-Stokes breathing.

\begin{tabular}{|c|c|c|c|}
\hline & Normal breathing & $\begin{array}{l}\text { Cheyne-Stokes } \\
\text { breathing }\end{array}$ & $P$ value \\
\hline Number & 60 & 87 & \\
\hline Age (years) & $60 \pm 14.5$ & $66 \pm 10.2$ & 0.01 \\
\hline Gender $(\mathrm{F} / \mathrm{M}=n)$ & $15 / 45$ & $14 / 73$ & NS \\
\hline $\mathrm{ICM}, n(\%)$ & $19(32)$ & $34(39)$ & NS \\
\hline BMI $\left(\mathrm{kg} / \mathrm{m}^{2}\right)$ & $24.6 \pm 2.9$ & $25.1 \pm 2.6$ & NS \\
\hline NYHA class & $2.2 \pm 0.8$ & $2.6 \pm 0.7$ & 0.01 \\
\hline $\mathrm{EF}(\%)$ & $33 \pm 8.5$ & $29 \pm 8.3$ & 0.01 \\
\hline Creatinine (mg/dl) & $1.08 \pm 0.3$ & $1.25 \pm 0.4$ & 0.01 \\
\hline $\mathrm{Hb}(\mathrm{g} / \mathrm{dl})$ & $13.2 \pm 1.2$ & $12.8 \pm 1.5$ & NS \\
\hline $\mathrm{AF}, n(\%)$ & $7(13)$ & $15(18)$ & NS \\
\hline Paced rhythm, $n(\%)$ & $5(9)$ & $7(8)$ & NS \\
\hline ACEi/ARBs, $n(\%)$ & $51(85)$ & $75(86)$ & NS \\
\hline Beta-blockers, $n(\%)$ & $41(74)$ & $59(71)$ & NS \\
\hline Spironolactone, $n(\%)$ & $31(56)$ & $51(61)$ & NS \\
\hline Digoxin, $n(\%)$ & $23(43)$ & $28(37)$ & NS \\
\hline Diuretic, $n(\%)$ & $44(81)$ & $65(87)$ & NS \\
\hline Workload (Watt) & $87 \pm 33$ & $71 \pm 29$ & 0.04 \\
\hline peakVO $_{2}(\mathrm{~mL} / \mathrm{min} / \mathrm{kg})$ & $13.4 \pm 4$ & $11.3 \pm 3.8$ & 0.02 \\
\hline $\mathrm{PETCO}_{2}(\mathrm{~mm} \mathrm{Hg})$ & $37.9 \pm 3.8$ & $33.1 \pm 4.2$ & 0.001 \\
\hline $\mathrm{ANP}(\mathrm{ng} / \mathrm{L})^{*}$ & $66.4(18-103)$ & $136.2(57-230)$ & 0.001 \\
\hline $\mathrm{BNP}(\mathrm{ng} / \mathrm{L})^{*}$ & $64.1(21-202)$ & $284(99-510)$ & 0.001 \\
\hline NT-proBNP (ng/L)* & $448.5(147-1599)$ & $2575(814-3320)$ & 0.001 \\
\hline PRA (ng/mL/h)* & $1.65(0.37-5)$ & $1.78(0.4-4.4)$ & NS \\
\hline Aldosterone (ng/L)* & $156(109-242)$ & $150(88-191)$ & NS \\
\hline Insulin (ng/L) & $12 \pm 8.1$ & $12 \pm 10.6$ & NS \\
\hline Cortisol (ng/L) & $168 \pm 74$ & $164 \pm 62$ & NS \\
\hline Epinephrine (ng/L)* & $31(10-42)$ & $35(19-52)$ & NS \\
\hline Norepinephrine $(\mathrm{ng} / \mathrm{L})^{*}$ & $438(331-681)$ & $588(395-939)$ & 0.01 \\
\hline fT3 (ng/L) & $2.4 \pm 0.1$ & $2.3 \pm 0.1$ & NS \\
\hline fT4 (ng/L) & $12.5 \pm 0.4$ & $13.7 \pm 0.5$ & NS \\
\hline TSH (ng/L)* & $1.5(0.68-1.8)$ & $1.5(0.76-2.7)$ & NS \\
\hline
\end{tabular}

F: females; M: males; ICM: ischaemic cardiomyopathy; BMI: body mass index; NYHA: New York Heart Association; EF: left ventricular ejection fraction; AF: atrial fibrillation; ACEi: angiotensin-converting enzyme inhibitors; ARBs: angiotensin-receptor blockers; peak $\mathrm{VO}_{2}$ : peak oxygen consumption; $\mathrm{PETCO}_{2}$ : end-tidal carbon dioxide partial pressure; ANP: atrial natriuretic peptide; BNP: brain natriuretic peptide; NT-proBNP: $\mathrm{N}$-terminal part of the pro-peptide of BNP; PRA: plasma renin activity; fT3: free triiodothyronine; fT4: free tetraiodothyronine; TSH: thyroid stimulating hormone; $\mathrm{Hb}$ : hemoglobin. Values are expressed as mean $\pm \mathrm{SD}$ for normally distributed variables, and $\left(^{*}\right)$ as median (25th-75th percentile) for non-normally distributed variables; $\%$ and (number of cases) are presented for binary variables.

ventilatory efficiency during exercise (expressed as slope of the ventilation vs $\mathrm{VCO} 2$ relation in its linear part), plasma level of BNP, NT-proBNP, norepinephrine, plasma renin activity. Among the univariate predictors (age, ejection fraction, atrial fibrillation, daytime CS, NYHA functional class, serum creatinine, plasma level of BNP and NTproBNP), only the two with highest level of significance (EF and plasma level of NT-proBNP were then considered for multiple logistic regression analysis) due to the limited number of events during follow-up [14].

Values are presented as mean \pm standard deviation (SD), or median and interquartile range (for variables with non-
Table 2

Predictors at univariate and multivariate analysis of daytime CS respiration

\begin{tabular}{llll}
\hline & Odds ratio & $95 \%$ C.I. & $P$ value \\
\hline Univariate analysis & & & \\
PETCO $_{2}$ & 0.74 & $0.62-0.88$ & 0.001 \\
peakVO $_{2}$ & 0.87 & $0.76-0.99$ & 0.030 \\
EF & 0.95 & $0.91-0.99$ & 0.013 \\
Workload & 0.98 & $0.96-0.99$ & 0.018 \\
Age & 1.05 & $1.02-1.08$ & 0.002 \\
BNP & 1.78 & $1.36-2.32$ & 0.000 \\
NT-proBNP & 1.87 & $1.41-2.48$ & 0.000 \\
ANP & 2.07 & $1.36-3.16$ & 0.001 \\
NYHA & 2.10 & $1.29-3.42$ & 0.003 \\
Norepinephrine & 2.36 & $1.25-4.46$ & 0.008 \\
Creatinine & 4.21 & $1.48-12.01$ & 0.007 \\
& & & \\
Multivariate analysis & & $1.01-1.13$ & 0.034 \\
Age & 1.06 & $1.13-3.30$ & 0.017 \\
NT-proBNP & 1.93 & & \\
\hline
\end{tabular}

CI: confidence intervals; $\mathrm{PETCO}_{2}$ : end-tidal carbon dioxide partial pressure; peak $\mathrm{VO}_{2}$ : peak oxygen consumption; EF: left ventricular ejection fraction; BNP: brain natriuretic peptide; NT-proBNP: N-terminal part of the propeptide of BNP; ANP: atrial natriuretic peptide; NYHA: New York Heart Association.

normal distribution) and $P$ values $<0.05$ were considered significant.

\section{Results}

\subsection{Prevalence of daytime breathing disorders}

Daytime CS (Fig. 1) was found in $87(59 \%)$ patients: central hypopneas were found in $47(32 \%)$ and central apneas in $40(27 \%)$ patients. All apneas and hypopneas were identified as having a central origin (characterized by simultaneous absence of both $\mathrm{CO} 2$ flow and chest/ abdominal respiratory activity).

Table 3

Independent predictors at univariate and multivariate analysis of cardiac mortality.

\begin{tabular}{llll}
\hline & Hazard ratio & $95 \%$ C.I. & $P$ value \\
\hline $\begin{array}{l}\text { Univariate analysis } \\
\text { peakVO }\end{array}$ & & & \\
EF & 0.83 & $0.70-0.98$ & 0.027 \\
Age & 0.89 & $0.83-0.95$ & 0.001 \\
NYHA & 1.06 & $1.02-1.26$ & 0.042 \\
CS & 2.29 & $1.29-4.07$ & 0.005 \\
BNP & 3.21 & $1.23-8.41$ & 0.017 \\
NT-proBNP & 3.38 & $1.84-6.22$ & 0.000 \\
AF & 4.16 & $2.10-8.25$ & 0.000 \\
& 4.69 & $1.70-12.91$ & 0.003
\end{tabular}

Multivariate analysis

NT-proBNP $\quad 2.98$

$1.35-6.56$

0.007

PeakVO ${ }_{2}$ : peak oxygen consumption; EF: left ventricular ejection fraction; NYHA: New York Heart Association classification; CS: daytime CheyneStokes respiration; BNP: brain natriuretic peptide; NT-proBNP: N-terminal part of the pro-peptide of BNP; AF: atrial fibrillation. 
In the subset of patients who underwent nocturnal polysomnography, the presence of respiratory disorders at nighttime was accurately predicted by concomitant daytime CS (AUC 0.821, $P<0.01$ at receiver operating characteristic analysis, sensitivity $75 \%$, specificity $75 \%$ ). Patients with significant nocturnal CS (Respiratory Disorder Index $>20$ ) showed lower $\mathrm{PETCO}_{2}(31.9 \pm 5$ vs $35.9 \pm 5 \mathrm{~mm} \mathrm{Hg}$, $P=0.025)$, higher BNP [540 (229-881) vs 159 (55-319) $\mathrm{ng} / \mathrm{L}, P=0.001]$ and NT-proBNP level [3001 (1802-3547) vs 1238 (282-3352), $P<0.001$ ], and worse NYHA functional class $(2.9 \pm 0.5$ vs $2.5 \pm 0.5, P=0.003)$.

\subsection{Clinical features, neurohormonal evaluation and breathing abnormalities}

Presence of CS was significantly associated with a more severe clinical impairment (i.e. NYHA classification), age, reduced EF and functional capacity, lower levels of resting $\mathrm{PETCO}_{2}$, and renal impairment. Patients with CS presented significantly higher plasma norepinephrine and cardiac natriuretic peptide levels (Fig. 2), as compared to patients with normal breathing, whereas no differences between the two groups were found for aldosterone, PRA, epinephrine and thyroid hormone plasma levels (Table 1).

\subsection{Predictors of daytime respiratory disorders}

At univariate analysis, the occurrence of daytime CS was predicted by age, low EF, low $\mathrm{PETCO}_{2}$ level at rest, increased level of serum creatinine, plasma norepinephrine, ANP, BNP, and NT-proBNP (Table 2). However, at multivariate analysis age and plasma NT-proBNP level were the only independent predictors of CS; in particular, plasma NT-proBNP level resulted the best predictor (AUC $0.735, P<0.001$, cut-off

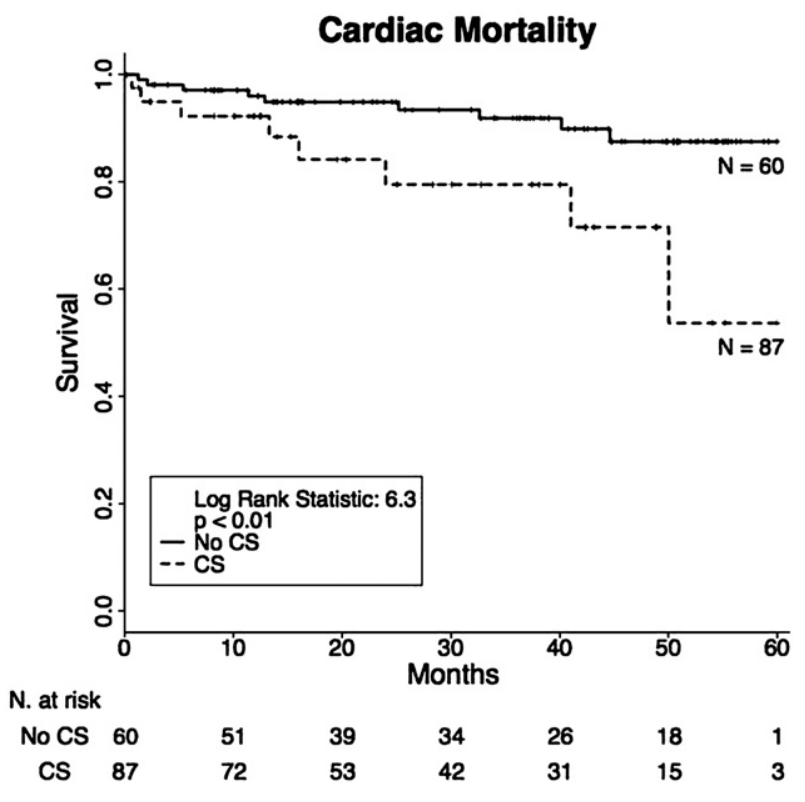

Fig. 3. Survival among 147 patients with chronic heart failure, according to the presence of daytime Cheyne-Stokes (CS) respiration. value $634 \mathrm{pg} / \mathrm{mL}$, specificity $61 \%$, sensitivity of $85 \%$, positive predictive value $76 \%$, negative predictive value $73 \%$ ).

\subsection{Identification of risk factors for cardiac death}

Median follow-up was 33 months (range 6-2000 days), during which 17 cardiac deaths occurred (3 sudden deaths and 14 due to congestive heart failure). Four non-cardiac deaths were excluded from the analysis.

At the univariate analysis, the following variables were predictive of cardiac death (Table 3): daytime CS (Fig. 3), increased plasma level of BNP and NT-proBNP (hazard ratios refer to a risk increase for one unit increment of their natural logarithm), age, presence of atrial fibrillation, higher NYHA class, EF. At the multivariate analysis, only NTproBNP was an independent predictor of mortality.

\section{Discussion}

Our study indicates that central apneas and hypopneas occur in a high percentage of CHF patients at daytime, despite treatment with neurohormonal antagonists. Moreover, daytime CS is associated with adrenergic activation and overexpression of cardiac natriuretic hormones. Among all risk factors for daytime CS at univariate analysis, the concentration of plasma NT-proBNP was the best independent predictor of breathing abnormalities.

The prevalence of daytime CS in the present study is lower, as compared to earlier reports [4,8], likely due to improvement in pharmacological treatment during the last decade, in which beta-blockers had not been administered systematically [15]. The pathogenesis of CS in CHF is multifactorial and involves deregulation of negative feedbacks secondary to enhanced chemoceptive sensitivity [1619], increased circulation time [18], increased filling pressures, and decreased functional residual capacity [20]. The combination of these factors elicits an excessive ventilatory response, lowering the difference between circulating levels of carbon dioxide and the apneic threshold, and thus favouring central apneas [21].

Daytime CS was significantly associated with more severe clinical impairment, reduced left ventricular EF and functional capacity, and lower levels of resting $\mathrm{PETCO}_{2}$. The latter finding suggests a common pathogenesis for daytime and nocturnal CS. Indeed, the absence of obstructive apneas in awake patients at daytime confirms previous observations $[4,8]$ : in awake status there is adequate stimulation of dilator muscles to maintain upper airway patency [22].

We also observed adrenergic activation in patients with daytime CS (likely associated with activated chemoreflex and CS-related oxyhemoglobin desaturation and hypoxia) [23], which in turn may contribute to ventricular arrhythmias and adverse prognosis [24,25], as well as increased expression of both atrial and B-type natriuretic peptides. Cardiac natriuretic hormone production is elicited by increased cardiac filling pressures [11], which have been 
associated with presence of sleep-related central apneas [26], sympathetic activation [11], and hypoxia [27].

Male gender, atrial fibrillation, age $>60$ years, and hypocapnia during wakefulness have been previously defined as risk factors for central sleep apneas by Sin, who, however, had not studied neurohormonal indices [13]. In our study, we found that, in addition to age and hypocapnia, left ventricular systolic dysfunction, NYHA class, functional capacity, and NT-proBNP were associated with CS. The ability of NT-proBNP level to predict daytime CS follows previous reports on increased BNP concentration in patients with sleep-related respiratory disorders [28,29], confirming the close relationship between cyclical respiratory pattern, haemodynamic overload and hypoxemic status [11,20].

Finally, our findings establish the value of resting daytime $\mathrm{CS}$ in the prediction of cardiac death in CHF, paralleling the observation by Corrà [30] on either sleep or exertional periodic breathing, and by La Rovere [31], though only plasma level of NT-proBNP resulted as independent prognosticator.

In a recent study by Brack et al., [32] CS during $>10 \%$ of daytime was an independent predictor of death, after adjusting for BNP, age, and NYHA class. However, the authors enrolled only 60 patients and employed a long-term recording device without taking into account patient-to-patient variations in postural changes, speech and physical daily activity.

The limited sample size and the low cardiac event rate did not allow us to evaluate the association of daytime CS with specific causes of cardiac death. Furthermore, a thorough echocardiographic evaluation of left ventricular diastolic function was available only in a portion of study population, thus precluding its use in the overall analysis.

In conclusion, $\mathrm{CS}$ at daytime is easily and detectable in an objective way in CHF patients. It is a frequent manifestation and is associated with adrenergic activation and increased natriuretic hormone plasma level. Daytime recording of respiratory pattern, especially in the presence of increased NT-proBNP levels, could discriminate patients with breathing abnormalities. Further studies are necessary to evaluate specific therapies, targeting chemoceptive feed-back and adrenergic activation. Respiratory [33] and aerobic physical training [34], in particular, may have the potential to improve life quality and life expectancy in this particular subset of CHF patients.

\section{References}

[1] Javaheri S. Sleep-related breathing disorders in heart failure. In: Mann DL, editor. Heart failure: a companion to Braunwald's Heart Disease. Philadelphia: Saunders; 2004. p. 471-87.

[2] Yamashiro Y, Kryger MH. Review: sleep in heart failure. Sleep 1993;16: 513-23.

[3] Skobel E, Norra C, Sinha A, Breuer C, Hanrath P, Stellbrink C. Impact of sleep-related breathing disorders on health-related quality of life in patients with chronic heart failure. Eur J Heart Fail 2005;7:505-11.

[4] Mortara A, Sleight P, Pinna GD, et al. Abnormal awake respiratory patterns are common in chronic heart failure and may prevent evaluation of autonomic tone by measures of heart rate variability. Circulation 1997;96:246-52.
[5] Van de Borne P, Oren R, Abouassaly C, Anderson E, Somers VK. Effect of Cheyne-Stokes respiration on muscle sympathetic nerve activity in severe congestive heart failure secondary to ischemic or idiopathic dilated cardiomyopathy. Am J Cardiol 1998;15:432-6.

[6] Lanfranchi PA, Braghiroli A, Bosimini E, et al. Prognostic value of nocturnal Cheyne-Stokes respiration in chronic heart failure. Circulation 1999;99:1435-40.

[7] Sin DD, Logan AG, Fitzgerald FS, Liu PP, Bradley TD. Effects of continuous positive airway pressure on cardiovascular outcomes in heart failure patients with and without Cheyne-Stokes respiration. Circulation 2000;4(102):61-6.

[8] Ponikowski P, Anker SD, Chua TP, et al. Oscillatory breathing patterns during wakefulness in patients with chronic heart failure: clinical implications and role of augmented peripheral chemosensitivity. Circulation 1999; 100:2418-24.

[9] Andreas S, Hagenah G, Moller C, Werner GS, Kreuzer H. CheyneStokes respiration and prognosis in congestive heart failure. Am J Cardiol 1996;7:1260-4.

[10] Emdin M, Passino C, Prontera C, et al. Cardiac natriuretic hormones, neuro-hormones, thyroid hormones and cytokines in normal subjects and patients with heart failure. Clin Chem Lab Med 2004;42:627-36.

[11] Clerico A, Recchia FA, Passino C, Emdin M. Cardiac endocrine function is an essential component of the homeostatic regulation network: physiological and clinical implications. Am J Physiol, Heart Circ Physiol 2006;290:H17-29.

[12] Clerico A, Emdin M. Diagnostic accuracy and prognostic relevance of the measurement of cardiac natriuretic peptides: a review. Clin Chem 2004;50:33-50.

[13] Sin DD, Fitzgerald F, Parker JD, Newton G, Floras JS, Bradley TD. Risk factors for central and obstructive sleep apnea in 450 men and women with congestive heart failure. Am J Respir Crit Care Med 1999;160:1101-6.

[14] Katz MH. Multivariable analysis: a primer for readers of medical research. Ann Intern Med 2003;138:644-50.

[15] Tamura A, Kawano Y, Naono S, Kotoku M, Kadota J. Relationship between beta-blocker treatment and the severity of central sleep apnea in chronic heart failure. Chest 2007;131:130-5.

[16] Javaheri S. A mechanism of central sleep apnea in patients with heart failure. N Engl J Med 1999;341:949-54.

[17] Naughton M, Benard D, Tam A, Rutherford R, Bradley TD. Role of hyperventilation in the pathogenesis of central sleep apneas in patients with congestive heart failure. Am Rev Respir Dis 1993;148:330-8.

[18] Hall MJ, Xie A, Rutherford Ret, Ando S, Floras JS, Bradley TD. Cycle length of periodic breathing in patients with and without heart failure. Am J Respir Crit Care Med 1996;154:376-81.

[19] Solin P, Roebuck T, Johns DP, Walters EH, Naughton MT. Peripheral and central ventilatory responses in central sleep apnea with and without congestive heart failure. Am J Respir Crit Care Med 2000;162:2194-200.

[20] Wasserman K, Zhang YY, Gitt A, et al. Lung function and exercise gas exchange in chronic heart failure. Circulation 1997;96:2221-7.

[21] Hanly P, Zuberi N, Gray R. Pathogenesis of Cheyne-Stokes respiration in patients with congestive heart failure. Am J Respir Crit Care Med 2002;165:1245.

[22] Kuna S, Remmers JE. Anatomy and physiology of upper airway obstruction. In: Kryger MG, Roth T, Dement MC, editors. Principles and practice of sleep medicine. 3rd ed. Philadelphia: WB Saunders; 2000. p. 840-58.

[23] Coy TV, Dimsdale JE, Ancoli-Israel S, Clausen J. Sleep apnoea and sympathetic nervous system activity: a review. J Sleep Res 1996;5:42-50.

[24] Staniforth AD, Sporton SC, Early MJ, Wedzicha JA, Nathan AW, Schilling RJ. Ventricular arrhythmia, Cheyne-Stokes respiration, and death: observations from patients with defibrillators. Heart 2005;91:1418-22.

[25] Javaheri S, Shukla R, Zeigler H, Wexler L. Central sleep apnea, right ventricular dysfunction, and low diastolic blood pressure are predictors of mortality in systolic heart failure. J Am Coll Cardiol 2007;49: 2028-34.

[26] Solin P, Bergin P, Richardson M, Kaye DM, Walters EH, Naughton MT. Influence of pulmonary capillary wedge pressure on central apnea in heart failure. Circulation 1999;30:1574-9. 
[27] Goetze JP, Gore A, Moller CH, Steinbruchel DA, Rehfeld JF, Nielsen LB Acute myocardial hypoxia increases BNP gene expression. FASEB J 2004;18:1928-30.

[28] Christ M, Sharkova Y, Fenske H, et al. Brain natriuretic peptide for prediction of Cheyne-Stokes respiration in heart failure patients. Int J Cardiol 2007;116:62-9.

[29] Carmona-Bernal C, Quintana-Gallego E, Villa-Gil M, SanchezArmengol A, Martinez-Martinez A, Capote F. Brain natriuretic peptide in patients with congestive heart failure and central sleep apnea. Chest 2005; 127:1667-73.

[30] Corra U, Pistono M, Mezzani A, et al. Sleep and exertional periodic breathing in chronic heart failure: prognostic importance and interdependence. Circulation 2006;113:44-50.
[31] La Rovere MT, Pinna GD, Maestri R, et al. Clinical relevance of shortterm day-time breathing disorders in chronic heart failure patients. Eur J Heart Fail 2007;9:949-54.

[32] Brack T, Thüer I, Clarenbach CF, et al. Daytime Cheyne-Stokes respiration in ambulatory patients with severe congestive heart failure is associated with increased mortality. Chest 2007;132: $1463-71$.

[33] Spicuzza L, Gabutti A, Porta C, Montano N, Bernardi L. Yoga and chemoreflex response to hypoxia and hypercapnia. Lancet 2000;356:1495-6.

[34] Passino C, Severino S, Poletti R, et al. Aerobic training decreases Btype natriuretic peptide expression and adrenergic activation in patients with heart failure. J Am Coll Cardiol 2006;47:1835-9. 